\title{
Cultivating the Installed Base: The Introduction of e-Prescription in Greece
}

\author{
Polyxeni Vassilakopoulou and Nicolas Marmaras
}

\subsection{Introduction}

E-prescription was introduced in Greece during times of financial turbulence with the aim to enhance control over pharmaceutical expenditure and also, to improve doctor-pharmacy collaboration and patient safety and to support evidence-based policy development. In that sense, the introduction of e-prescription is not yet another technology project but rather, a socio-technical intervention with infrastructural nature. In this chapter we explore the national e-prescription service's surprisingly swift deployment. Specifically, we identify how a series of pragmatic tactical decisions allowed building upon a "good-enough" installed base by exploiting its latent potential without perpetuating all of its weaknesses. Furthermore, we show how hedging against obsolescence was practiced through continuously addressing exogenous shifts in the installed base. Finally, we point to the pivotal role of the technical architecture implemented for enabling installed base cultivation. A combination of novel technological affordances, standards and architectural patterns made possible the development of a technical solution which supports openness, evolvability and scalability.

In our study we position e-prescribing within the overall Greek health system and we describe how the new electronic service evolved to inscribe specific

\footnotetext{
P. Vassilakopoulou $(\triangle)$

University of Agder, Postboks 422, 4604 Kristiansand, Norway

University of Oslo, Oslo, Norway

e-mail: polyxenv@uia.no

N. Marmaras

National Technical University of Athens, Heroon Polytechniou 9,

15780 Zografou, Athens, Greece

e-mail: marmaras@central.ntua.gr
} 
prescribing policies, to provide clinical decision support, and to facilitate the processes and roles of policy and financing stakeholders. Our case description spans the period from 2010 to 2015 .

\section{Data Collection}

To (re)construct e-prescription's trajectory: extensive documents' review including legislation and guidelines, policy documents and strategic plans, press releases (from Social Security Funds, the Ministry of Health, and IDIKA), public consultation documents, presentation documents from various professional and academic events, posts in professional electronic forums, articles in specialised press and journals.

To develop an understanding of the e-prescription solution: on-site observations of e-prescription use in pharmacies. The observations were repeated in 2 month intervals. Additionally, we studied the user manuals for pharmacists and doctors.

To elicit practitioners' perspectives: seven semi-structured interviews.

The remaining of the chapter is structured as follows: in Sect. 7.2 we present an overview of the Greek healthcare system and the situation with respect to information systems, in Sect. 7.3 we present the rationale for the introduction of e-prescription in Greece, we provide an overview of the e-prescription service and we describe its evolution over time, then, in Sect. 7.4 we discuss the relationship to the installed base. Finally, in Sect. 7.5 we provide some concluding remarks.

\subsection{Healthcare in Greece}

\subsubsection{Overview of the Greek Healthcare System}

Healthcare delivery in Greece is based on both public and private providers (mainly in primary care and diagnostic tests). The Greek national health system (ESY) was established after a major healthcare reform in 1983 with the aim to guarantee universal healthcare coverage for all (universal healthcare rights are stipulated by the Greek Constitution). Public health provision is coordinated by seven Health Regions that are supervised by the Ministry of Health. Secondary healthcare is provided by public and private hospitals and clinics. Primary healthcare is provided through rural health centers and provincial surgeries in rural areas, the outpatient departments of regional and district hospitals in urban areas and contracted doctors with private practices (OECD 2013a). Unlike what is common in many other European countries, Greek residents do not have to register with General Practitioners (GPs) and GPs do not have a gate-keeping role. Individuals can access the entire spectrum of specialists for consultations and can be directly referred by them for reimbursable 
tests and examinations. Because of the structure of healthcare provision, and the lack of a GP referral system, free choice of provider is a key characteristic of the system. The ownership of pharmacies is limited to pharmacists. Pharmacies are licensed by the government on the basis of criteria for population coverage and distance from the nearest existing pharmacy.

Key health indexes for the Greek population are good. In the 2000 report by the World Health Organization on health systems' performance, the Greek healthcare system was ranked 14th worldwide in terms of overall performance and 11th on level of health (World Health Organization 2000). During recent years, healthcare cost containment has been the main Government's concern. This concern is induced both by the rise in healthcare services demand (due to the aging population, the increase of patients living with chronic conditions and citizens' pressures for increasing the supply of quality healthcare services) but also, by the need to address the ongoing public debt crisis.

As in nearly all European countries, the public sector is the main source of healthcare financing. Financing is provided mainly by social security funds (although out-of-pocket-payments and direct health financing from the national budget of the central government are also significant). Most of the funds are public entities (legal persons governed by public law), and while they are autonomous, they operate under the control of central government. The funds cover both pensions and healthcare for particular socio-professional groups (i.e. there are different funds for farmers, public servants, etc.) on the basis of personal contributions but the state also contributes to their financing. The number of funds was brought down from 130 to 13 in 2008 (OECD 2013a) and there is further consolidation underway. For healthcare, the aim is to merge all healthcare coverage schemes (that relate to different funds) to a single one. On March 2011, the healthcare schemes for farmers, freelance non-professional workers and public servants were subsumed by the scheme for non-public sector salaried employees (IKA). All together came under the umbrella of a new organisation named "National Organisation for Health Services Provision" (EOPYY, incorporated with Law 3918/2011) which started operating in 2012. EOPYY is still being expanded to cover the beneficiaries of all other social insurance funds and is gradually becoming a single public buyer of healthcare goods and services. Figure 7.1 provides an overview of the main actors involved in healthcare regulation, provision and financing.

Aggregate public spending for health is moderate compared to EU and OECD averages (OECD 2013b). Although the overall expenditure is moderate, the statistics indicate room for improvement especially within pharmaceuticals where the annual expenditure both per capita and as a share of the Gross Domestic Product is high (about 40\% more than the EU average) (OECD 2013b). This high expenditure has been a key concern for the Government also because health goods are predominantly financed by public funds (74\% of expenditure is publicly financed in Greece while only $54 \%$ in Europe as an average (OECD 2013b)). 


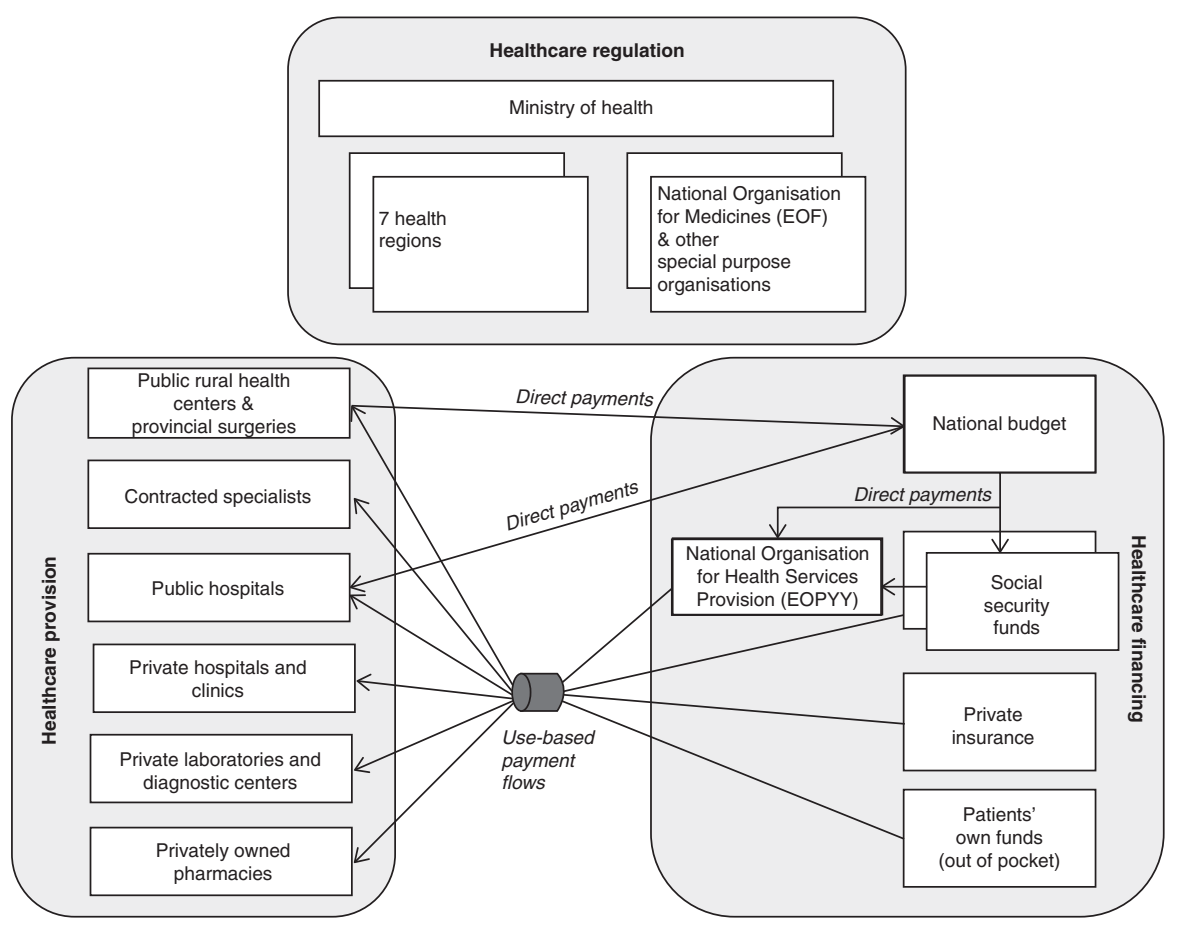

Fig. 7.1 Greek healthcare: regulation, financing and service provision (as of 2015)

\subsubsection{Information Systems in Greek Healthcare}

Initial efforts for the introduction of information systems within Greek healthcare date back to the 1980s (Fragidis and Chatzoglou 2011). Nevertheless, although a series of national plans were drafted and pursued (e.g. most recently, the national 2002-2006 Action Plan "ICT in healthcare" and the national eHealth roadmap 2006-2015), the progress achieved has not always been significant.

Notwithstanding the delays, there has been a clear positive trend in information systems' use over the years. Practically all pharmacies use information systems. Within primary care, as of 2013, $99 \%$ of GP practices had computers in the consultation room as opposed to only $66 \%$ back in $2007,99 \%$ of practices were connected to the internet or a dedicated GP network and 24\% had their own website (European Commission DG Communications Networks Content and Technology 2013). The electronic storage of medical patient data is relatively common among GPs although it is not universally exercised: around $70 \%$ of GPs store electronically the medical history of their patients and more than $60 \%$ register electronically their clinical notes, symptoms and ordered tests (idem). Health information exchange is much less developed among GPs (idem): only around 22\% receive laboratory reports electronically and around $20 \%$ exchange medical patient data with other healthcare 
providers and professionals (excluding prescriptions), electronic interactions with patients are also limited (27\% of GPs).

As of 2013, practically all hospitals (99\%) used computer systems; billing management $(90 \%)$ and discharge letters (76\%) were the most common hospital applications (European Commission Joint Research Centre Institute for Prospective Technological Studies (JRC-IPTS) 2014). Hospital-wide electronic health record systems shared by all clinics were used in around half of the hospitals (52\%) another $13 \%$ used multiple local systems, while $35 \%$ had no health record system in place (idem). Only $24 \%$ of hospitals exchanged health information (excluding prescriptions) with entities outside the hospital (e.g. other hospitals, external specialists, GPs) (idem).

Medical data exchange has been impeded by the lack of a single personal identifier for all Greek residents up till recently (the obligatory social security number - AMKA was only introduced in October 2009 (Greek Ministry for Labour 2012)), the delays in establishing a secure network (the secure network "Syzefxis" that connects all public entities including healthcare was only initiated in 2004, became operational in 2006 and it is still under development although it has now achieved significant coverage) and the multitude of solutions with different logics and limited standardization (Emmanouilidou and Burke 2012; Bogdanos et al. 2008).

\subsection{The Introduction of E-Prescription}

\subsubsection{Rationale for E-Prescription and Key Milestones}

Greece introduced e-prescription to enhance control over pharmaceutical expenditure, to improve doctor-pharmacy collaboration and patient safety and to capture data required for evidence-based policy development. The aspired benefits were clearly set-out in the law that provides the legal basis for e-prescription (Law 3892/2010). The year when the e-prescription law passed (year 2010) the Greek economy was facing a severe public debt crisis which captured global attention. In return for loans from the International Monetary Fund and European Institutions, the Greek government agreed to accelerate reforms including structural reforms of the healthcare sector and the introduction of new electronic tools. The strong financial motivation behind the e-prescription initiative is demonstrated by its inclusion in May's 3rd 2010 "Memorandum of Economic and Financial Policies" between Greece and the International Monetary Fund and subsequently in the "Hellenic National Reform Programme 2011-2014" issued on April 2011.

The introduction of e-prescription was swift: development started in 2010, a pilot was run in October of the same year and the official launch was on January 24th 2011 (Table 7.1). By the fall of 2011 around 40\% of prescriptions were covered, and by fall 2013 almost full coverage was reached (Papanikolaou 2013). E-prescription was one of the initiatives that contributed to the reduction of the total pharmaceuticals' expenditure by approximately 33\% between the years 2009 
Table 7.1 Greek e-prescription: key facts

\begin{tabular}{|c|c|c|c|c|}
\hline \multirow{4}{*}{\multicolumn{2}{|c|}{$\begin{array}{l}\text { Function } \\
\text { Guide prescribing } \\
\text { behaviour, support } \\
\text { registration and circulation } \\
\text { of prescription and } \\
\text { dispensing information }\end{array}$}} & Users & \multicolumn{2}{|c|}{ Temporal evolution } \\
\hline & & \multirow{2}{*}{$\begin{array}{l}\text { General practitioners } \\
\text { and specialists in } \\
\text { primary care, private } \\
\text { and public hospitals }\end{array}$} & \multicolumn{2}{|c|}{ Initiated in 2010} \\
\hline & & & \multirow{2}{*}{\multicolumn{2}{|c|}{$\begin{array}{l}\text { Launched in January } 2011 \\
\text { (pilot October 2010) }\end{array}$}} \\
\hline & & Pharmacists & & \\
\hline & & $\begin{array}{l}\text { Reimbursing } \\
\text { authorities }\end{array}$ & \multirow{2}{*}{\multicolumn{2}{|c|}{ Almost full coverage ( $98 \%$ ) by 2013}} \\
\hline & & $\begin{array}{l}\text { Public health policy } \\
\text { makers }\end{array}$ & & \\
\hline & $\begin{array}{l}\text { Fall 2010 } \\
\text { (pilot) }\end{array}$ & Fall 2011 & Fall 2012 & Fall 2013 \\
\hline Pharmacists & $\sim 8,500$ & $\sim 8,500$ & $\sim 10,800$ & $\sim 10,800(98 \%$ of total $)$ \\
\hline Doctors & $\sim 4,100$ & $\sim 10,100$ & $\sim 37,500$ & $\sim 41,000(90 \%$ of total $)$ \\
\hline $\begin{array}{l}\text { Prescriptions } \\
\text { (monthly) }\end{array}$ & $\sim 8000$ & $\sim 2,500,000$ & $\sim 4,500,000$ & $\begin{array}{l}\sim 6,000,000 \\
(98 \% \text { of total })\end{array}$ \\
\hline
\end{tabular}

and 2011 (Greek e-Government Centre for Social Security 2011). In the next sections we present the trajectory followed starting with a brief presentation of the situation before the introduction of the new electronic service.

\subsubsection{Information Handling Before the Introduction of E-Prescription}

Before the introduction of e-prescription prescribing was supported by "prescription booklets" issued by Greek social security funds. These booklets were kept by the patients and used during their interactions with doctors and pharmacists. The booklets were personalised: they contained a photo, identity information such as name, birth date, address, registration id (for the fund's internal registry), national tax id and a unique identification number per booklet. Each booklet contained fifty double-sided prescription pages and their carbon copies (a white coloured original and a yellow coloured copy). Each prescription page had on the one side fields to be used by the prescribing doctor (including doctors' information, diagnosis, drugs description and quantity) and by the dispensing pharmacist (including pharmacist information, drugs' cost and patient's cost share) and on the other side a template for attaching identifying adhesive labels from the packaging of the drugs dispensed. These labels are mandatory for all drugs circulating in Greece. Drugs carry a serial number that identifies each pack uniquely. Serial numbers are used for preventing reimbursement fraud and monitoring consumption and expenditure. The booklet format was defined in 1998 (presidential decree 82A/1998) and revised in 2008 to include the national insurance number (AMKA) and a barcode. Figure 7.2 presents the standard prescription template that was in use before the introduction of e-prescription. 

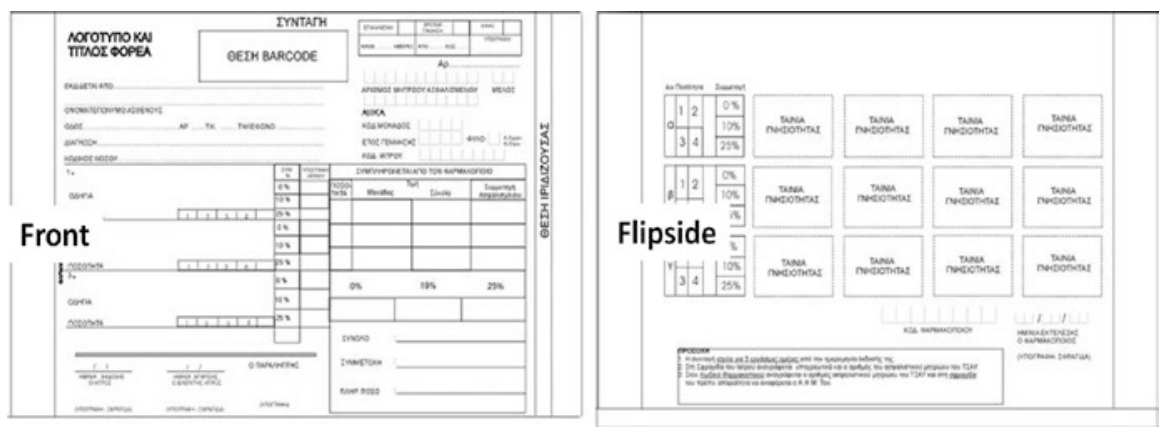

Fig. 7.2 Paper prescription template

Patients carried with them their prescription booklets when visiting a doctor. Doctors would use a page for prescribing drugs, sign and stamp the page and hand the booklet back to patients. Afterwards, patients could visit any pharmacy, hand the booklet to the pharmacist who would then complete the remaining fields on the front of the prescription page, sign and stamp, tear off the page (the yellow copy remained in the booklet), fetch the prescribed drugs from storage, detach the identification labels from the drug packages and attach them to the page, handover the drugs to the patient and collect payment (patient's share of cost). Periodically, pharmacists would send to social security funds lists with filled prescriptions attaching the white prescription pages in order to be reimbursed. The booklet's yellow pages served as records for the medication history of each patient.

For social security funds, processing prescriptions' data required resources and a dedicated infrastructure. For instance, IKA (the largest social security fund) conceptualised a project in 2005 for the electronic processing of the white prescription pages received. A request for proposals was published in 2007 and a contract was signed in 2009 for the development of a scanning and processing system and its initial operation for 2 years (with a total cost of approximately 6 million Euro). The system was in place in April 2010 and made possible the scanning, checking and clearing of 2.5 million prescriptions per month (IKA 2009; Hararis 2011). The systems that social security funds have developed for scanning, checking and clearing prescriptions are part of the overall prescriptions' installed base and had to be eventually linked to the e-prescription solution (see also Sect. 7.3.4).

\subsubsection{Information Handling After the Introduction of E-Prescription}

A graphical representation of the Greek e-prescription service is provided in Fig. 7.3. Web-based access is provided to prescribing doctors and pharmacists. Access is controlled at the user level (registered users go through a username and 


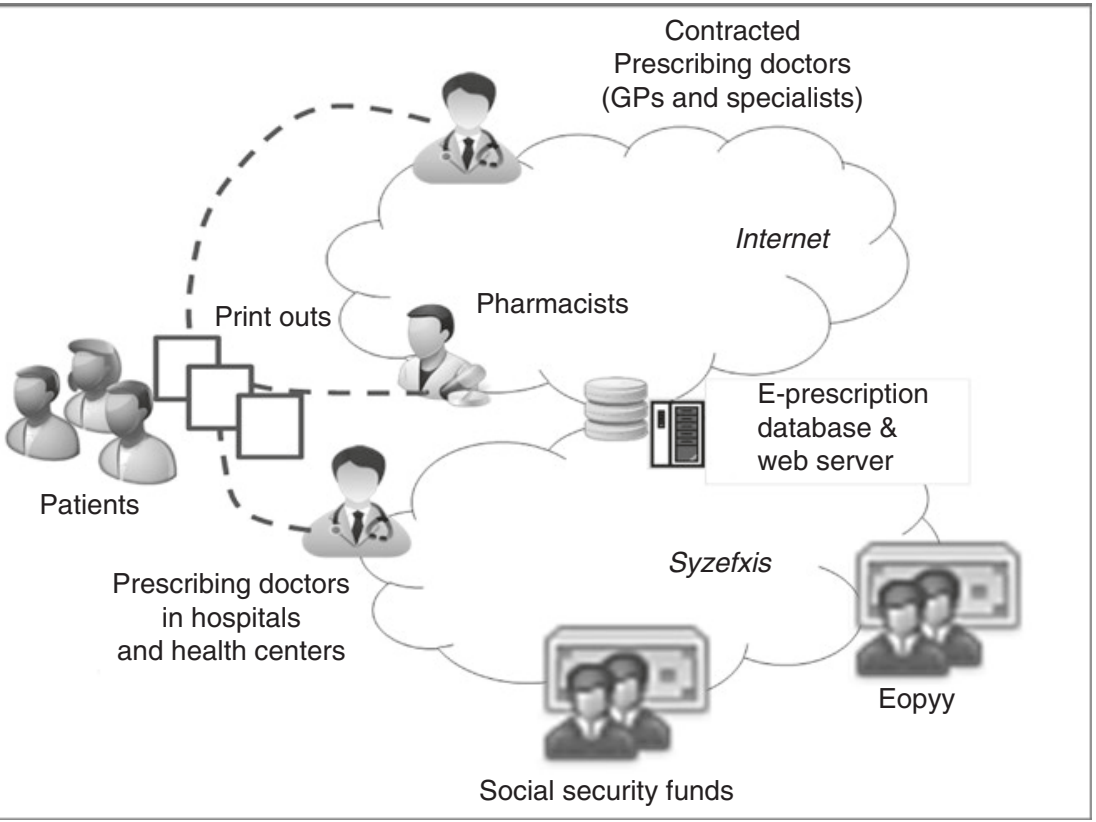

Fig. 7.3 E-prescription in Greece

password identification process) and a central repository of all prescriptions is maintained nationally. Hospitals access the service over the closed secure network Syzefxis, all other healthcare users use their private internet connections. Prescribing doctors register key information (including the patient's name and social security number, the diagnosis encoded according to ICD-10, and the medications prescribed) and then, print a summary page which is handed to the patient. Patients can visit any pharmacy in order to obtain prescribed medications. Pharmacists take the printed prescription summary page and scan the barcode to retrieve the prescription from the national central repository (alternatively they can type). Before delivering medications, pharmacists scan the medication packages' barcodes which are then matched to prescription details. In case of mismatch an error message appears on the screen and processing cannot be completed.

As with the previous fully paper-based process, pharmacists detach package labels and attach them to the prescription printout before handing over medications to patients and collecting payment (patient's share of cost). The bottom part of the printout contains designated positions for placing the labels (Fig. 7.4). Periodically, pharmacists send to reimbursement authorities lists with the prescriptions they filled attaching the printouts that include the identification labels of the medications dispensed. Doctors can use e-prescription for retrieving the full prescriptions' history per patient (pharmacists do not have access to this functionality). Patients do not have direct access to e-prescription data. 


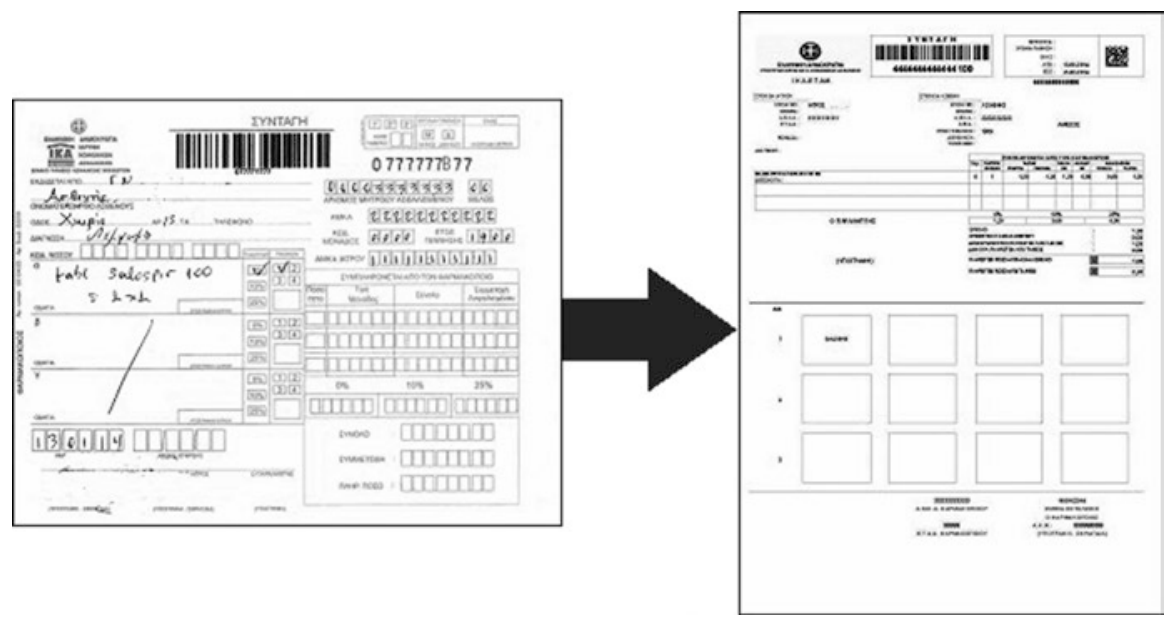

Fig. 7.4 Prescriptions in Greece: from booklet pages to printouts

The e-prescription solution is hosted and maintained by the Greek e-Government Centre for Social Security (IDIKA) which has developed it in-house. IDIKA is supervised and controlled by the Greek Ministry of Labour, Social Security and Welfare; it is mainly financed by the social security funds and is responsible for the implementation of information and communication technology within the social security sector. E-prescribing was initially piloted in October 2010 for patients covered by one specific social security fund (the fund for self-employed workers - OAEE). For the piloting of the service almost all pharmacists were enrolled along with doctors contracted by the specific fund (see also Table 7.1). In January 2011, the service was officially launched and three more social security funds were added: the fund for non-public sector salaried employees (IKA) which is the largest in the country, the fund for farmers (OGA) and the fund for public servants (OPAD). In 2012 a number of additional funds were included: in April, the fund for seafarers (NAT), in May, the fund for bank employees (TAITEKO), in November, the fund for employees in the mass media (ETAP MME) and in December, the fund for lawyers, engineers, doctors, dentists, veterinarians and pharmacists (ETAA). Each addition necessitated information exchange with additional fund-specific registries. The establishment of the new "National Organisation for Health Services Provision" (EOPYY) which started operating in 2012 and gradually assimilated the healthcare insurance schemes of multiple funds (as described in Sect. 7.2.1) helped in the establishment of common rules but the different funds retained their separate registries.

The software development for e-prescription was initially contracted to external providers and the first versions launched were not developed in-house. Two lowbudget contracts were signed with two relatively small software houses (the total value including contract extensions for accommodating additional social security 
funds was approximately 200,000 euro (АГГЕ $\Lambda$ ОПО' $\Lambda$ OY 2012)). At the beginning of 2012 IDIKA started the in-house development of a new e-prescription solution which was successfully launched in May 2012 (Sfyroeras 2012b). The new in-house development aimed to remedy a series of issues: slow response times and concerns about scalability, reliability and usability. It also provided the opportunity for expansions and service improvements in a flexible incremental way. The inhouse development was an interim solution which became necessary as the procurement of the fully-fledged solution through a public tendering process (which was initiated in 2010) was delayed due to administrative procedures (Pangalos and Asimakopoulos 2015).

\subsubsection{System Evolution}

The in-house version of e-prescription was launched in May 2012 and included enhanced functionality. For instance, it supported the automatic retrieval of basic patient information, it provided doctors the option to use multiple affiliations (i.e. doctors working both for a private practice and a private clinic), it simplified the repeat prescriptions' process and offered improved search functionalities. This was the start of a continuous effort for incremental improvements and extensions.

\section{Connections and Extensions}

The initial versions of e-prescription were only accessible via web browsers. There was no connectivity to the EPRs already in use by doctors or to pharmacy information systems (PISs). A major improvement was the publishing of Application Programming Interfaces (APIs) that allowed connectivity with doctors' and pharmacists' systems. In the spring of 2012 IDIKA initiated discussions with system providers for the APIs that were under development. The APIs were initially tested

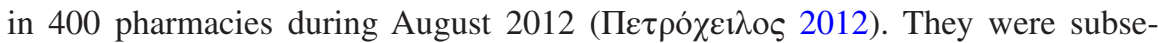
quently used by multiple system providers connecting the majority of pharmacies (by the end of 2012). In 2015 the APIs for doctors' EPRs were launched (Tagaris 2015). The web service APIs developed adhere to REST architectural constraints (RESTful APIs) and to the Clinical Document Architecture (CDA) markup standard to specify the encoding, structure and semantics of exchanged documents. The introduction of the APIs and their exploitation by third party system providers not facilitated everyday work for pharmacists and doctors that could now conclude their tasks without having to use multiple applications. Figure 7.5 provides an overview of the key architectural components for e-prescription (adapted from Asimakopoulos (2012)). The figure depicts also the link with the scanning and processing systems (for prescription printouts and attached medication labels) of the social security funds (Scan SFF).This was an additional extension implemented during the same period. The e-prescription team collaborated also with the European project epSOS for cross-border interoperability of summaries of electronic health records and 


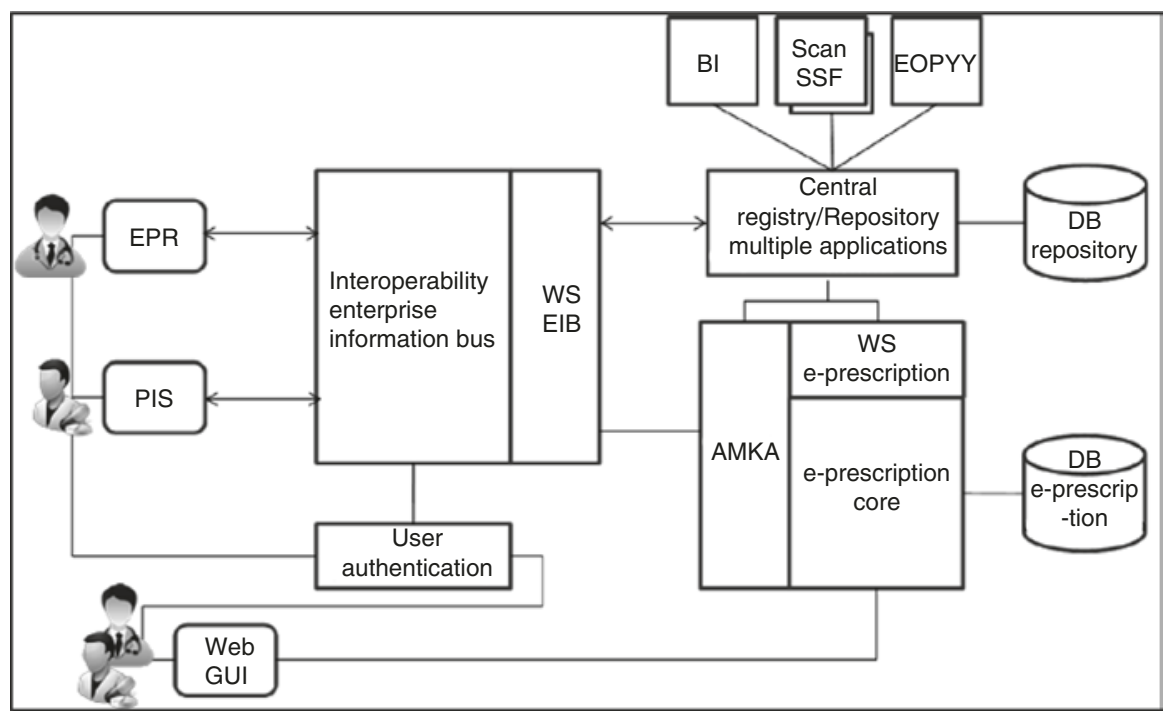

Fig. 7.5 Key architectural components of the Greek e-prescription

e-Prescriptions. Hence, the system can process epSOS friendly prescriptions for cross-border healthcare.

The publishing of APIs and the subsequent adaptation of the local EPRs and PISs, made possible for doctors and pharmacists to prescribe and dispense medications without having to shift between the web interface and their local systems. Still, doctors that needed not only to prescribe medications but also to order diagnostic tests (e.g. diagnostic imaging and blood tests) had to access an additional system (named e-diagnosis) via a web interface. The electronically supported process for test ordering is similar to the process for electronic drug prescribing: doctors register key information (including the patient's name and social security number, the diagnosis encoded according to ICD-10, and the tests ordered) and then print a summary page which is handed to the patient. Patients can visit public or private contracted laboratories and diagnostic centers for performing the tests. The e-diagnosis system for test ordering was initially launched in October 2010 (for patients covered by the social security fund for public servants - OPAD) and was developed and maintained by a private software company. In May 2011, it was decided to simplify use by applying a common user authentication scheme for both e-prescription and e-diagnosis but the two systems were kept separate. After the successful launch of the in-house version of e-prescription, IDIKA decided to extend its functionality by including test ordering. In January 2013, a new extended version of e-prescription was launched that made possible for doctors to prescribe drugs and order tests from within the same environment. 
As medications are reimbursed mainly by social security funds, information about patients' affiliation with a specific fund is needed for prescribing medications (to apply specific reimbursement rules). When e-prescription was first introduced the funds were maintaining multiple electronic registries for their members and several of those registries were incomplete (for example, most registries did not include information about children that are fund beneficiaries when one of their parents is a fund member) (Sfyroeras 2012c). Before the introduction of e-prescription, doctors and pharmacists would find information about a patient's affiliation by simply looking at the prescriptions' booklet. The booklets also contained information on the status of patients as insured members (status relates to the payment of dues to the fund - benefits can only be claimed if dues are paid). For the digital process both affiliation and status information needed to be electronically available. IDIKA was already responsible for the national registry for social security (AMKA-EMAE $\Sigma$ ) that contains national social security numbers (AMKA) and basic information per individual (name, date of birth, parents' names). The AMKA registry did not contain information about the status of individuals' relationship with particular funds. In an initiative parallel to e-prescription, a new system named ATLAS that includes a new national registry for all healthcare beneficiaries was developed and launched by IDIKA in 2014. ATLAS links multiple registries and supports the flow and storage of information on insurance status and social insurance contributions. ATLAS is not dedicated to healthcare, it is also meant to support the calculation of pensions. This new system was linked to e-prescription in the summer of 2014.

\section{Inscriptions of Administrative Rules and Clinical Knowledge}

Overall, several rules related to reimbursement are inscribed to e-prescription. To start with, the electronic service was designed to replicate the simple constraints of the paper-based system that was previously in place. Up to three different medications can be included in one prescription (see also Fig. 7.4); in case that more are needed, separate additional prescriptions have to be issued. Furthermore, specific rules for medication quantities are also in place - rules differ for chronic patients, specific types of medications etc. Since June 2012, substance-based prescribing (instead of naming pharmaceutical products) was electronically imposed. The classification of active ingredients of medications is based on the ATC international classification system. This new rule (substance-based prescribing) was subsequently relaxed so e-prescribing was readjusted. Recently, (September 2015) the rule was reintroduced in the system after yet another change in the reimbursement regulations. The rules for patients' cost-sharing are also inscribed in the electronic solution (and are being updated each time they change). The general rule is that patients contribute $25 \%$ of medications' cost but there are many special patient and/or therapy-specific categories for which the contribution is $10 \%$ or even $0 \%$ (e.g. chronic patients, pregnant women, patients with transplanted organs, etc.). Additionally, there are rules for the maximum amounts that patients may pay. Specific constraints on 
what can be prescribed by doctors according to their specialty are also implemented. Additionally, there are specific rules for prescribing doctors that limit the number of prescriptions that can be issued and define upper bounds (caps) for the total permitted cost per prescription (since 2013 different methods for calculating caps were applied based on simple data analytics e.g. by taking into account the prescribing history of individual doctors or specific specialties and geographic areas).

The rules inscribed to e-prescription are not only related to costs and reimbursements. Therapeutic prescribing protocols for a series of conditions (i.e. diagnosis-based prescribing guidelines) have also been electronically implemented. Practically, this means that e-prescribing gradually extended to become a decision support tool for doctors. The protocols include medication of "first choice", secondary medications, alternative therapies and rare cases. The medication options are described on the basis of active substance. These protocols are developed by specially appointed committees for condition categories defined by the National Organisation for Medicines (EOF) and are approved by the Central Health Council (KESY). A total of 160 protocols were developed and approved on October 2011. The first protocols were launched within e-prescription in October 2013 (for osteoporosis) and since then, their number has been continuously increasing. Up to September 201515 different protocols were implemented (e.g. for dyslipidaemia, diabetes, arterial hypertension and rheumatological conditions) while there are several more under development with the aim to be launched before the end of 2015 (for dementia, Parkinson disease, epilepsy, chronic obstructive pulmonary disease, chronic bronchitis, asthma).

\section{Working Around Complications in National Plans}

The national eHealth roadmap 2006-2015 included a plan for the introduction of smart cards in healthcare. The smart cards would be used both for identification and authorisation purposes (for patients and healthcare providers) and also for storing data (administrative identification, clinical emergency data, prescriptions and insurance status) (Angelidis et al. 2010). Small-scale experimentations with smart cards for healthcare have been taking place in Greece since the 1990s (Karounou and Vassilakopoulos 1995). However, the plan for national level deployment of smart cards for health has not been materialised till today. The exploration of the whole spectrum of issues that impede the national deployment of smart cards for health in Greece is outside the scope of this chapter but we can briefly mention issues related to the cost and complexity of extending the existing physical infrastructure to include card readers, the need for large-scale organisational and regulatory adaptations and discussions/disputes around data security and data ownership. Nevertheless, as smart health cards are part of the national plan the introduction of e-prescription was linked to the use of the cards and that was clearly stated when the consultation process for the development of the new electronic prescription services was initiated back in 2010 (the use of PKI-based smart cards was part of the requirements). 
IDIKA was also involved in small-scale experimentations with smart cards. Specifically, a pilot was launched in 2012, in the prefecture of Corinthia were 2500 individuals insured by the social security fund for municipal employees (TYDKY) were provided with personalized smart cards. The pilot was discussed in public by IDIKA management: the stated aim was to explore and prepare for national scale implementation; it was also announced that IDIKA was planning to provide 60.000 smart cards to healthcare providers (covering all prescribing doc-

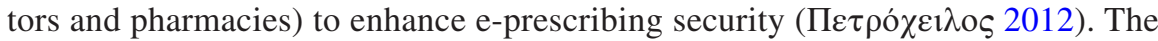
use of smart cards for e-prescription was never scaled-up but the pilot showed the preparedness of the system to accommodate the national strategy for smart cards in healthcare.

The government's intention for nationwide deployment of smart cards in healthcare has been recently reconfirmed and the current plan is to use the cards both for healthcare and social benefits (Greek Ministry of the Interior and Administrative Reform 2015). Still, the necessary arrangements for nationwide deployment are not in place while the e-prescription service is deployed nationally. Given the current situation, the much awaited security enhancement of e-prescription is being currently implemented with the introduction of USB tokens for healthcare providers (launched in June 2015). While till recently access healthcare providers were accessing e-prescription using their user name and password, with the introduction of the tokens authentication is performed by the combination of the password and the USB token (two-way authentication).

The new authentication component is an outcome of the large-scale e-prescription project that was awarded to a consortium of companies. As already mentioned the in-house developed system is a makeshift solution that was put in place for the interim period required to implement the system acquired through a public procurement process. This process was initiated with a public consultation on the design and implementation of the e-prescribing system (February-March 2010). This was followed by another public consultation which was specific to the implementation stages for e-prescription (April 2010). Subsequently, the tendering documents were made available for public consultation in April 2011. After that, a two-step tendering process was initiated. An open call for the project (budget Euro 24,6 million duration 36 months) was published in September 2011, four consortia were pre-selected (March 2012) and subsequently three of them submitted proposals (August 2012). The proposals were evaluated through a lengthy process that culminated in the contract award (June 2014). The value of the contract was significantly lower than the original budget (approx. 40\% lower) and the duration was set to 18 months. The new e-prescription solution was still under development at the time of writing.

The overall system evolution described in this section is graphically represented in Fig. 7.6. The figure depicts key milestones for the system-in-use and also for the public procurement process (for the fully-fledged solution) which has been running in parallel. 


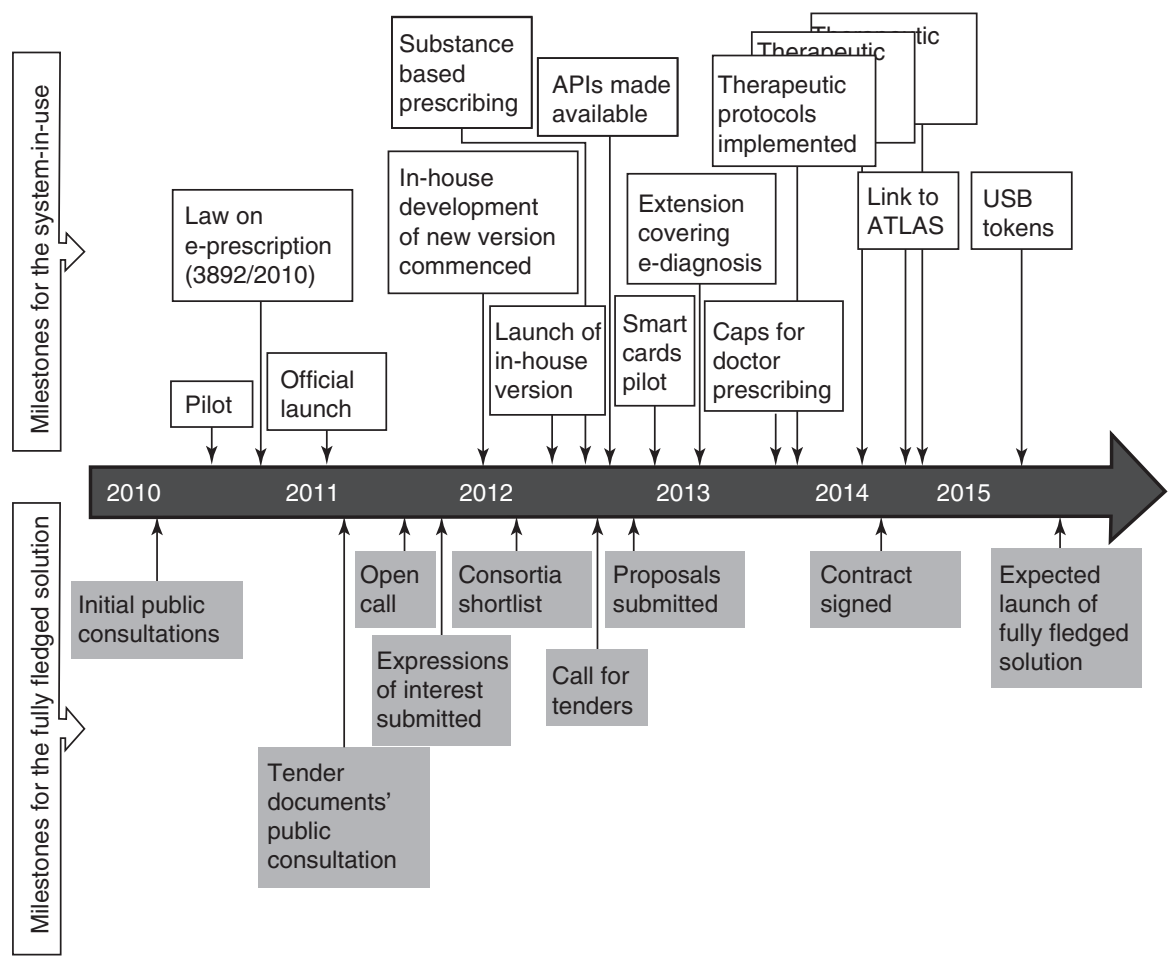

Fig. 7.6 Key milestones for the Greek e-prescription case

\subsection{Discussion: Relationship to the Installed Base}

\subsubsection{Building Upon an Installed Base That Is "Good Enough" Without Perpetuating All Weaknesses}

Gaps in the backbone of the country's information infrastructure caused difficulties to previous eHealth initiatives. Efforts to harmonize Greek healthcare with European "best practices" have repeatedly failed to deliver expected results and some of them were abandoned altogether (Economou 2010). Heath data exchange was impeded by the lack of a single personal identifier for all Greek residents (each social security fund used its own registry with its own identifiers) and the lack of a secure network to connect healthcare facilities. A number of recent initiatives with infrastructural nature filled some of these gaps and created a more favourable environment for the initiation of e-prescription. A new secure network (Syzefxis) supports connections among public institutions and provides gateways to the internet. A single national social security number (AMKA) was introduced in October 2009. Furthermore, computer-based information systems were present in practically all hospitals, primary healthcare units and pharmacies although as recently as 10 years 
ago this was not the case (see also Sect. 7.2.2). Additionally, standards for information codification like the International Classification of Diseases (ICD), the Anatomical Therapeutic Chemical (ATC) classification and HL7 Clinical Document Architecture (CDA) were already mature and readily available. E-prescription was built upon these enabling components and would have been challenged without them. Moreover, software architectural styles that allow client-server separation of concerns and simplify modular implementation (such as REST) were established. So, the development of the software capitalised on relevant technical knowledge and experience.

The installed base included also a complicated arrangement of multiple social security funds and national actors including the Ministry for Health, the Ministry for Labour Social Security and Welfare, the National Organisation for Medicines, Doctors and Pharmacists Associations. All these actors were involved by setting rules and providing datasets required for digitising the prescribing process. The Chief Executive Officer of IDIKA stated in an interview in 2012 that the main challenges faced were not related to the technical development but rather to the coordination of all involved actors (Sfyroeras 2012a). He also stated that the lack of interoperability among systems and the absence of a national registry for the beneficiaries of healthcare were assessed as showstoppers by some participants during the early stages of the initiative. Moreover, he pointed to other key components that were missing when the development of e-prescription started: lack of a full list of medications available in Greece (not just a list of approved medications), lack of a common identifier for medications, lack of a unified doctors' registry (multiple registries in place).

Although a number of key components were missing, the new system was not merely built upon the installed base perpetuating all of its weaknesses. Instead, several initiatives were taken to fill some of the gaps. For example, it would have been possible to circumnavigate problems with the national medications' list by allowing users to enter free-text medication descriptions. This would facilitate the circulation of messages between doctors and pharmacists but would be an inefficient solution for monitoring prescribing practices. The lack of standardised medications' lists is a problem in many other countries including USA were free-texting of e-prescription medications is common (Dhavle and Rupp 2014). However, in the Greek case, it was decided not to follow such an approach, instead, comprehensive lists were created and maintained, new registries were put in place, and new connections were implemented.

Overall, a pragmatic approach was adopted: some gaps were filled while others were worked around. For instance, for almost 5 years access control to e-prescription was rudimentary. Authentication was performed by means of user name and password. The implementation of mechanisms for two-factor authentication required the deployment of a physical infrastructure (smart cards or usb tokens) which was costly and logistically demanding. Hence, it was initially postponed and was eventually implemented in 2015. 


\subsubsection{Handling Continuous Exogenous Shifts in the Installed Base}

The situation within the installed base kept changing during the 5-years trajectory not only as a result of initiatives triggered by the need to put e-prescription in place but also because multiple initiatives related to wider reforms within healthcare took place. The institutional environment changed with the establishment of the National Organisation for Health Services Provision (EOPYY) which started operating in 2012 and is gradually becoming a single public buyer of healthcare goods and services. Additionally, a number of social security funds were merged. Furthermore, the distribution of roles and responsibilities among existing actors changed. For example, since October 2012 the price lists for medications are issued by the National Organisation of Medicines and not by the General Secretariat for Trade. The e-prescription service had to adapt to all these changes. Moreover, new potentially useful infrastructural components were created after the initial launching of e-prescription. For instance, therapeutic prescribing protocols were made available in 2011 and were subsequently progressively included in e-prescription.

Although several of the installed base changes were planned and known in advance, e-prescription would not be developed by taking them for granted as it is not uncommon to experience delays or even radical twists in national plans (a good example is the situation with smart cards for health where there is practically no significant advancement till today). Consequently, all decisions had to be based on the situation at hand while maintaining openness to accommodate changes. Part of the overall uncertain situation was the public procurement process for a fullyfledged system which was under way but without any certainty about the timing of the delivery. Hence, there was a need to adapt swiftly and cost-effectively since it was already known that the system in use would be replaced at some point. What was pivotal for this continuous effort to develop and maintain e-prescription through adaptations was the clear ownership and dedication by a single institution (IDIKA). This institution took the seemingly paradoxical decision to develop in-house at the beginning of 2012 a new version (even though the fully-fledged solution was already planned), replacing the one that was in place and was already reaching its limits (see also Sect. 7.3.3).

\subsubsection{Installed Base Cultivation vs. Specifications-Driven Development}

The tactics described in the two previous sections can be summarised as pragmatic exploitation and expansion of a "good enough" installed base, and continuous adaptation to exogenous shifts within this base. This can be characterised as a "cultivation" approach. In that approach, the installed base is not considered as a given and stable foundation for further developments that can be fully planned. Instead, the 
dynamics of this base are acknowledged and hence, interventions are attempted in an active interplay with it (Ciborra 1992; Ciborra and Hanseth 1998). Such an approach towards the installed base necessitates a requisite technical design that supports openness, evolvability and scalability. These were key characteristics of the new version of the system that was developed and launched in 2012. Specifically, the architectural configuration of the new system (Fig. 7.5) allowed loose coupling among components, offered the possibility for continuous new releases and supported component modifiability to meet changing needs.

A cultivation approach to the installed base entails incremental and evolutionary development which is drastically different to the conventional specificationsdriven approach that was followed in the past for national systems. For instance, the tax authorities' system was launched after 7 years of systematic design and implementation efforts (Prasopoulou 2012), while the system for social security reached countrywide implementation after almost two decades of planning and multiple discontinuities in the design and development process (Avgerou and McGrath 2007). For the procurement of the fully-fledged system the specification-driven approach was also adopted and it would be interesting to know how e-prescription would turn out without the prior experience of cultivation for over 5 years.

\subsection{Concluding Remarks}

E-prescription played a key role for the establishment of new rules and norms disrupting existing practices within healthcare. The introduction of the new electronic service was legitimised by referring to the expected economic impact (Greek e-Government Centre for Social Security 2011; Sfyroeras 2012a; Vassilakopoulou and Marmaras 2015) and to obligations towards the International Monetary Fund and European Institutions. The need for cost containment was undisputed as expenditure on pharmaceuticals had reached very high levels: per capita pharmaceutical expenses in \$ purchasing power parities (PPP) rose from 461 in 2004 to 840 in 2009 (OECD 2015b). The government managed to reduce the annual bill for pharmaceuticals by $€ 1.8$ billion between 2009 and 2013 (OECD 2015a). This significant cost cutting cannot be attributed to e-prescription alone. It was the outcome of several concurrent measures some of which were related to e-prescription e.g. favouring the use of generic medicines via substance-based prescribing and introducing caps per prescribing doctor. Additional measures not related to e-prescription include a new reference pricing model that takes into account the three EU countries with lowest prices, and the renegotiation and reduction of pharmacy and wholesaler margins on reimbursed drugs (OECD 2013a; Siskou et al. 2014; Deloitte Centre for Health Solutions 2013). The sense of crisis certainly facilitated change nevertheless, this by itself is not sufficient. The overall outcome was made possible by a combination of institutional leverage, novel technological affordances, and pragmatic tactical decisions. 
Open Access This chapter is distributed under the terms of the Creative Commons AttributionNonCommercial 2.5 International License (http://creativecommons.org/licenses/by-nc/2.5/), which permits any noncommercial use, duplication, adaptation, distribution and reproduction in any medium or format, as long as you give appropriate credit to the original author(s) and the source, provide a link to the Creative Commons license and indicate if changes were made. The images or other third party material in this book are included in the work's Creative Commons license, unless indicated otherwise in the credit line; if such material is not included in the work's Creative Commons license and the respective action is not permitted by statutory regulation, users will need to obtain permission from the license holder to duplicate, adapt or reproduce the material.

\section{References}

Angelidis P, Giest S, Dumortier J, Artmann JH. Country brief: Greece. European Commission, DG Information Society and Media, ICT for Health Unit; 2010.

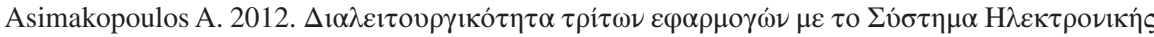

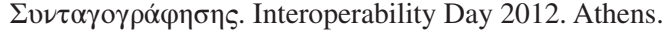

Avgerou C, McGrath K. Power, rationality, and the art of living through socio-technical change. MIS Q. 2007;31:295-315.

Bogdanos C, Lagouros M, Ekonomou L. 2008. Healthcare information systems in Greece: system and human integration. Commun Inform Technol:196-200.

Ciborra C. From thinking to tinkering: the grassroots of strategic information systems. Inf Soc. 1992;8:297-309.

Ciborra C, Hanseth O. 1998. Toward a contingency view of infrastructure and knowledge: an exploratory study. Proceedings of the international conference on Information systems. Assoc Inf Syst:263-72.

Deloitte Centre For Health Solutions. Impact of Austerity on European pharmaceutical policy and pricing. staying competitive in a challenging environment. Deloitte LLP, London; 2013.

Dhavle A, Rupp M. 2014. Towards creating the perfect electronic prescription. J Am Med Inform Assoc:22;e7-12.

Economou C. Greece: health system review. Health Syst Transit. 2010;12:1-180.

Emmanouilidou M, Burke M. A thematic review and a policy-analysis agenda of Electronic Health Records in the Greek National Health System. Health Policy;2012;109(1):31-37.

European Commission DG Communications Networks Content \& Technology. Benchmarking deployment of ehealth among general practitioners - final report - country profile: Greece. Luxembourg: Publications Office of the European Union; 2013.

European Commission Joint Research Centre Institute For Prospective Technological Studies (JRC-IPTS). European hospital survey: benchmarking deployment of ehealth services. Luxembourg: Publications Office of the European Union; 2014.

Fragidis LL, Chatzoglou PD. 2011. The use of electronic health record in greece: current status. Computer and Information Technology (CIT), 2011 IEEE 11th International Conference, IEEE, p. 475-80.

Greek E-Government Centre for Social Security 2011. Press release 10 Nov 2011.

Greek Ministry for Labour, S. S. A. W. 2012. Tı civol o AMKA (what is the social security number).

GREEK Ministry of the Interior and Administrative Reform. 2015. Press release: key points of the speech by Deputy Minister J. Katrougalos in the 5 th eGovernment Forum ( Г. КАTPOҮГКА $\Lambda$ Y $\Sigma$ TO 5o e-GOVERNMENT FORUM). Athens, 3 June 2015.

Hararis G.. IKA/EOPYY prescriptions processing system. Results after 20 months of productive operation. Annual Greek ICT Forum. Athens; 2011.

IKA. 2009. Press release on the system for automated prescriptions' management $-\Delta \varepsilon \lambda \tau$ ío Tútov $\gamma 1 \alpha$

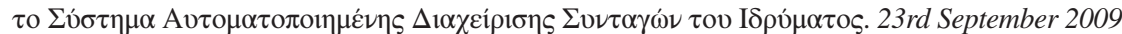
[Online]. Available: http://www.ika.gr/gr/infopages/press/20090923.cfm. Accessed 12 Sept 2015. 
Karounou V, Vassilakopoulos G. 1995. Plans and experiments with health cards in Greece. Studies in Health Technology and Informatics, 26: Health Cards '95, 229-32.

OECD. Greece: reform of social welfare programmes. Paris: OECD Publishing; 2013a.

OECD. Health at a glance 2013: OECD indicators. Paris: OECD Publishing; $2013 \mathrm{~b}$.

OECD. 2015a. Country note: how does health spending in Greece compare?

OECD. OECD health statistics 2015 - frequently requested data. In: OECD editor. July 2015, Last updated: 7 July 2015 ed; 2015b.

Pangalos G, Asimakopoulos D. 2015. The new Greek national e-prescription system: A powerful ehealth tool for improving services to citizens and administrative control. NHIBE 2015: New Horizons in Industry Business and Education. Skiathos.

Papanikolaou C. The Greek EU Presidency's plans in 2014: ehealth forum \& beyond. 3rd EU-US eHealth marketplace and conference. Boston; 2013.

Prasopoulou E. Addressing contextual influences during ICT innovation for public sector reform: the case of TAXIS. In: Papadopoulos T, Kanellis P, editors. Public sector reform using information technologies: transforming policy into practice. Hershey: IGI Global; 2012.

Sfyroeras V. 2012a. Interview with the CEO of IDIKA. Pharma and Health Business; p. 26-9.

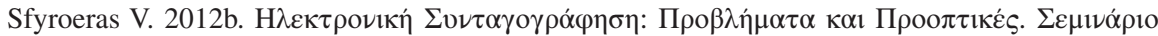

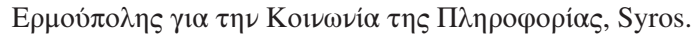

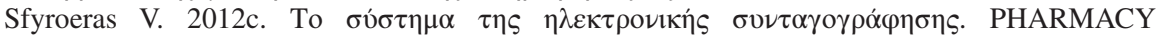
MANAGEMENT KAI EПIKOINSNIA. p. 68-9.

Siskou O, Kaitelidou D, Litsa P, Georgiadou G, Alexopoulou H, Paterakis P, Argyri S, Liaropoulos L. Investigating the economic impacts of new public pharmaceutical policies in Greece: focusing on price reductions and cost-sharing rates. Value Health Reg Issues. 2014;4:107-14.

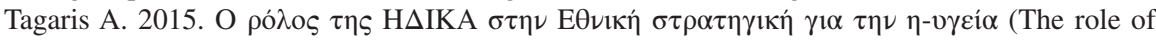

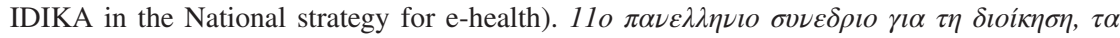

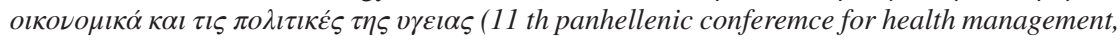
economics and policiew). Athens.

Vassilakopoulou P, Marmaras N. Investigating technology-induced transitions in healthcare: work practice adaptations within their overall context. Health Policy Technol. 2015;4:277-85.

World Health Organization. The world health report 2000. Health systems: improving performance. Geneva: Office of Publications, World Health Organization; 2000.

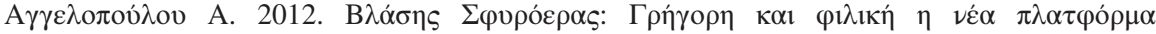

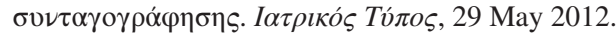

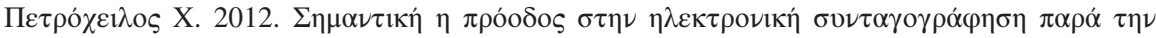

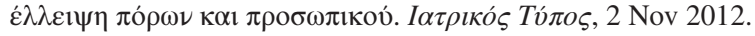

Open Access This chapter is distributed under the terms of the Creative Commons AttributionNonCommercial 2.5 International License (http://creativecommons.org/licenses/by-nc/2.5/), which permits any noncommercial use, duplication, adaptation, distribution and reproduction in any medium or format, as long as you give appropriate credit to the original author(s) and the source, provide a link to the Creative Commons license and indicate if changes were made.

The images or other third party material in this chapter are included in the chapter's Creative Commons license, unless indicated otherwise in a credit line to the material. If material is not included in the chapter's Creative Commons license and your intended use is not permitted by statutory regulation or exceeds the permitted use, you will need to obtain permission directly from the copyright holder.

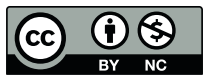

